\title{
Retreatment with bevacizumab in patients with gynecologic malignancy is associated with clinical response and does not increase morbidity
}

This article was published in the following Dove Press journal:

OncoTargets and Therapy

21 March 2014

Number of times this article has been viewed

\author{
Robin A Laskey' \\ Scott D Richard ${ }^{2}$ \\ Ashlee L Smith' \\ Jeff $F$ Lin' \\ Tiffany L Beck ${ }^{3}$ \\ Jamie L Lesnock' \\ Joseph L Kelley 3rd' \\ Alexander B Olawaiye' \\ Paniti Sukumvanich' \\ Thomas C Krivak ${ }^{4}$ \\ 'Division of Gynecologic Oncology, \\ Magee-Womens Hospital of UPMC, \\ Pittsburgh, ${ }^{2}$ Division of Gynecologic \\ Oncology, Drexel University College \\ of Medicine/Hahnemann University \\ Hospital, Philadelphia, ${ }^{3}$ Department \\ of Obstetrics, Gynecology and \\ Reproductive Sciences, Magee- \\ Womens Hospital of UPMC, \\ Pittsburgh, ${ }^{4}$ Division of Gynecologic \\ Oncology, Western Pennsylvania \\ Hospital, Pittsburgh, PA, USA
}

Correspondence: Thomas C Krivak Division of Gynecologic Oncology, Western Pennsylvania Hospital, 4800 Friendship Avenue, Pittsburgh,

PA I5244, USA

Tel +I 7247783033

Fax +I 7247783054

Email thomas.krivak@plzdocs.com
Purpose: Bevacizumab (Bev) is associated with improved progression-free survival in advanced epithelial ovarian cancer. The use of Bev in patients with gynecologic malignancy is increasing; however, little is known about cumulative toxicity and response in patients retreated with Bev. Our goal was to determine cumulative side effects and response in patients retreated with Bev.

Patients and methods: Women with recurrent gynecologic malignancy treated with Bev between January 2007 and March 2012 at a single institution were identified, including a subset who received Bev in a subsequent regimen. The primary outcome was Bev-associated toxicity, and the secondary outcome was response.

Results: Of 83 patients that received Bev for recurrent disease, 23 were retreated with Bev and four received Bev maintenance. Three patients (13\%) developed grade 3 or 4 hypertension; all had a history of chronic hypertension. One (4.3\%) patient developed grade 3 proteinuria, and one $(4.3 \%)$ developed an enterovaginal fistula. Four patients discontinued Bev secondary to toxicity. Toxicity was not related to the cumulative number of cycles. Twenty-six percent of patients responded to Bev retreatment. On univariate analysis, there was a significant $(P=0.003)$ overall survival advantage when the Bev-free interval was $>9$ months ( $95 \%$ confidence interval [CI] 4.9-43.7) compared to $\leq 9$ months (95\% CI 2.1-11.5), 24.3 months, and 6.8 months.

Conclusion: Retreatment of patients with recurrent gynecologic malignancy with Bev did not increase morbidity and was associated with treatment response. Physicians treating women with recurrent disease may consider a Bev-containing regimen even if prior regimen(s) included Bev. Future studies should prospectively evaluate the efficacy of this treatment strategy.

Keywords: bevacizumab, gynecologic malignancy, cumulative toxicity, treatment response

\section{Introduction}

Bevacizumab (Bev) (Avastin ${ }^{\circledR}$; Genentech, San Francisco, CA, USA) is a monoclonal antibody that targets vascular endothelial growth factor A (VEGF-A). It is the most extensively tested antiangiogenic agent, ${ }^{1}$ and is being increasingly incorporated into treatment regimens of patients with gynecologic malignancy. The results of Gynecologic Oncology Group (GOG)-218, a large Phase III clinical trial evaluating the efficacy of a Bev-containing regimen for frontline treatment of advanced epithelial ovarian cancer (EOC) were recently released. While there was no difference in overall survival, the trial did show a 4-month improvement in progression-free survival (PFS) in patients treated with a Bev-containing regimen compared to a nonBev-containing regimen. ${ }^{2}$ Similarly, the Gynecologic Cancer Intergroup International Collaboration on Ovarian Neoplasms (ICON)-7 trial showed a statistically-significant 1.7-month improvement in PFS in EOC patients treated with a Bev-containing regimen

submit your manuscript $\mid$ www.dovepress.con 
(24.1 months) compared to a non-Bev-containing regimen (22.4 months). ${ }^{3}$ Notably, in patients at high risk for progression, the difference in PFS was even greater between the Bev group and standard therapy group: 18.1 and 14.5 months, respectively. Following the publication of these clinical trials, the European Medicines Agency approved Bev, in addition to standard carboplatin and paclitaxel, as first-line treatment of ovarian cancer. ${ }^{4}$

Bev is also frequently utilized in the treatment of patients with recurrent gynecologic malignancy. The OCEANS (Ovarian Cancer Study Comparing Efficacy and Safety of Chemotherapy and Anti-Angiogenic Therapy in PlatinumSensitive Recurrent Disease) trial compared combination gemcitabine/carboplatin and Bev versus gemcitabine/ carboplatin and placebo for treatment of recurrent EOC. ${ }^{5}$ The Bev-containing arm showed improved PFS by 4 months when compared to the placebo (12.4 versus $8.4, P<0.0001)$. Additionally, those patients treated with Bev had better objective response rates $(78.5 \%$ versus $57.4 \%, P<0.0001)$ and longer duration of response. No new safety concerns were identified when Bev was used in the recurrent setting. There were higher rates of hypertension ( $\mathrm{HTN} ; 17.4 \%$ versus $<1 \%$ ) and proteinuria $(8.5 \%$ versus $<1 \%$ ), and two gastrointestinal perforations in the Bev-treatment arm. The improvement in PFS supports the use of Bev-containing regimens for the treatment of recurrent EOC.

Although the incorporation of Bev in treatment regimens for frontline and/or recurrent gynecologic malignancy has been increasing, few data exist regarding cumulative treatment-related toxicity and response in patients exposed to multiple chemotherapeutic regimens containing this biologic agent. Therefore, we sought to determine cumulative Bevassociated toxicity and treatment response rates in patients with recurrent gynecologic malignancy treated with multiple Bev-containing regimens.

\section{Patients and methods}

After obtaining institutional review board approval, we performed a single-institution retrospective review of women with recurrent gynecologic malignancy treated with a Bev-containing chemotherapeutic regimen between January 2007 and March 2012. Patients were identified via an institutional tumor registry and chemotherapy database. Eligibility criteria included age over 18 years and recurrent gynecologic malignancy treated with cytotoxic chemotherapy and Bev. Charts were reviewed to identify those patients who had received more than one Bev-containing regimen. These patients comprised the study population.
Patient information abstracted included age at diagnosis, primary site of disease, race, type of surgery (primary or interval), debulking status (suboptimal, optimal $<1 \mathrm{~cm}$ residual disease), International Federation of Gynecology and Obstetrics stage, histologic subtype, presence or absence of lymphovascular space invasion, peritoneal cytology, planned primary chemotherapy regimen, date of recurrent disease, regimen number in which the patient was first exposed to Bev, total number of cycles of Bev for each regimen, and whether or not the patient was maintained on Bev following completion of the cytotoxic component of each regimen, planned dose, and treatment schedule for each agent. Complications and clinical response for each regimen containing Bev were also recorded. Patients were typically followed with physical examination every 3 weeks while receiving treatment, and at least every 3 months when not actively receiving treatment. CA125 was usually obtained prior to each cycle; radiographic assessment of disease was obtained at the discretion of the treating physician, or as per clinical trial protocol. The lifetime number of cycles of Bev and disease status at last contact was recorded.

The primary outcome was Bev-associated toxicity, and the secondary outcome was treatment response. Toxicity was defined by the National Cancer Institute Common Terminology Criteria for Adverse Events version 4.0. ${ }^{6}$ Treatment response was defined by the treating physician using Response Evaluation Criteria In Solid Tumors ${ }^{7-9}$ and Rustin criteria. ${ }^{10-13}$ Complete response was defined as normalization of CA125 and disappearance of all target lesions on cross-sectional imaging; partial response was defined as a decrease in CA125 of more than $50 \%$ without complete normalization, and a decrease in the size but not complete disappearance of target lesions; progressive disease was defined as a doubling of CA125 from its nadir, an increase in the size of target lesions, or the development of new lesions on imaging; stable disease was defined as a decrease of CA125 of less than $50 \%$ maintained during the course of therapy and neither sufficient shrinkage to qualify for partial response nor sufficient increase to qualify for progressive disease.

Overall survival (OS) was estimated using the KaplanMeier method from three different time points to last contact or death: 1) initial surgery/diagnosis; 2) last diagnosis of recurrent disease; and 3) first day of last Bev regimen. Univariate analyses were performed using the log-rank test. Stratification was performed to control for number of interval chemotherapy regimens, as there were too few patients to construct a robust Cox proportional hazards model. Fischer's exact test was used to determine the significance of Bev-free 
interval and treatment response, with a $P$-value of $<0.05$ indicating significance.

\section{Results}

A total of 83 patients were identified that had received Bev for treatment of recurrent gynecologic malignancy. Of this group, a cohort of 23 patients was identified that had received more than one Bev-containing regimen, thus forming the study population. Patient demographics are shown in Table 1. Twenty women had EOC, two had uterine cancer, and one had a granulosa cell tumor of the ovary. The average age of diagnosis was 55.5 years. The vast majority of women were Caucasian. Only three patients had an early stage gynecologic malignancy: one patient was diagnosed with stage IC EOC, another with stage IIC EOC, and one with stage IA granulosa cell tumor. The remainder of the patients in this study had advanced-stage gynecologic malignancy.

Of the 23 patients retreated with Bev, seven had received three or more cytotoxic regimens containing Bev and four had received Bev maintenance following their first retreatment with a Bev-containing regimen. The initial and retreatment

Table I Patient demographics

\begin{tabular}{|c|c|}
\hline & $n=23$ \\
\hline Median age, years (range) & $55.5(34-78)$ \\
\hline \multicolumn{2}{|l|}{ Race } \\
\hline White & $21(91 \%)$ \\
\hline Black & $2(9 \%)$ \\
\hline \multicolumn{2}{|l|}{ Gynecologic malignancy } \\
\hline Epithelial ovarian cancer & $20(87 \%)$ \\
\hline Papillary serous & 13 \\
\hline Other & 7 \\
\hline Granulosa cell tumor & I (4\%) \\
\hline Uterine & $2(9 \%)$ \\
\hline Endometrial adenocarcinoma & I \\
\hline Carcinosarcoma & 1 \\
\hline \multicolumn{2}{|l|}{ Primary debulking status } \\
\hline Optimal & $12(52 \%)$ \\
\hline Suboptimal & $10(43 \%)$ \\
\hline Unknown & $\mathrm{I}(4 \%)$ \\
\hline \multicolumn{2}{|l|}{ Bev first line } \\
\hline Yes & $3(13 \%)$ \\
\hline No & $20(87 \%)$ \\
\hline \multicolumn{2}{|l|}{ Primary platinum sensitivity } \\
\hline Sensitive & $13(57 \%)$ \\
\hline Resistant & $4(17 \%)$ \\
\hline Refractory & $6(26 \%)$ \\
\hline Median regimen $\mathbf{n}$ first exposed to Bev, $\mathbf{n}$ (range) & $3(2-7)$ \\
\hline Median regimen retreated with Bev, $\mathbf{n}$ (range) & $4(3-10)$ \\
\hline Median cycles Bev w/retreatment (range) & $5(1-10)$ \\
\hline \multicolumn{2}{|l|}{ Median total lifetime cycles Bev } \\
\hline Without Bev maintenance (range) & $17(5-44)$ \\
\hline With Bev maintenance (range) & $35(25-59)$ \\
\hline
\end{tabular}

Abbreviation: Bev, bevacizumab.
Bev-containing regimens are shown in Table 2. The most common dosage and schedule of Bev was $15 \mathrm{mg} / \mathrm{kg}$ every 21 days, except for three patients treated with $10 \mathrm{mg} / \mathrm{kg}$ every 14 days. The median first exposure to Bev occurred with the third chemotherapy regimen (range 1-7) and retreatment with Bev occurred at a median fourth line (range 2-10). The median number of total cycles of Bev for patients that were retreated without maintenance therapy was 17 (range 5-44) compared to 35 (range 25-59) cycles for maintenance Bev. Five (20.8\%) patients had received 30 or more cycles of Bev.

Table 3 details the six patients that developed Bevassociated toxicities, including the toxicity, grade, cycle number when toxicity developed, treatment regimen, and toxicity management. Three patients $(13 \%)$ retreated with a Bev-containing regimen developed grade 3 or 4 HTN, and all patients had a previous diagnosis of chronic HTN. Treatment was ultimately discontinued due to toxicity in two of these patients. One patient (4.3\%), with stable disease and a history of chronic HTN and diabetes mellitus, developed grade 3 proteinuria after 25 cycles of Bev, requiring discontinuation. Finally, one patient (4.3\%) developed an enterovaginal fistula. Her history was significant for a vaginal cuff recurrence of ovarian cancer, treated with pelvic radiation. Six months after radiation, she again recurred and was started on a Bev-containing regimen. After 5 cycles, she developed an enterovaginal fistula requiring treatment discontinuation. The incidence of toxicity was not related to the number of cycles of Bev administered. Grade 3 or 4 toxicity developed at a median of nine cycles of Bev (mean 11.2 cycles, range 3-25). The remaining $74 \%$ of patients were able to complete their planned Bev-containing regimens without development of significant Bev-associated toxicity.

Primary adjuvant therapy for all patients was a platinum-based regimen. Fifty-seven percent of patients

Table 2 Bevacizumab (Bev)-containing regimens

\begin{tabular}{lll}
\hline & $\begin{array}{l}\text { Initial Bev- } \\
\text { containing } \\
\text { regimen } \mathbf{( n = 2 3 )}\end{array}$ & $\begin{array}{l}\text { Retreatment } \\
\text { Bev-containing } \\
\text { regimen } \mathbf{( n = 2 3 )}\end{array}$ \\
\hline Carboplatin/paclitaxel & 8 & 0 \\
Carboplatin/gemcitabine & 6 & $\mathrm{I}$ \\
Gemcitabine & 4 & 4 \\
Oxaliplatin & 3 & 3 \\
Oxaliplatin/docetaxel & $\mathrm{I}$ & 0 \\
Liposomal doxorubicin & 0 & 5 \\
Paclitaxel & 0 & $\mathrm{I}$ \\
Cytoxan & $\mathrm{I}$ & 3 \\
Etoposide & 0 & $\mathrm{I}$ \\
Everolimus & 0 & $\mathrm{I}$ \\
Single-agent Bev & 0 & 4 \\
\hline
\end{tabular}


Table 3 Bevacizumab (Bev)-associated toxicity

\begin{tabular}{|c|c|c|c|c|c|c|}
\hline Patient & Toxicity & Grade & $\begin{array}{l}\text { Cycle toxicity } \\
\text { developed }\end{array}$ & $\begin{array}{l}\text { Bev-containing } \\
\text { regimen }\end{array}$ & Treatment & Comments \\
\hline $\begin{array}{l}\text { I. 66-year-old with IIIC papillary } \\
\text { serous ovarian carcinoma }\end{array}$ & HTN & 3 & 9 & Gemcitabine & Held $\times$ I cycle & H/o chronic HTN \\
\hline $\begin{array}{l}\text { 2. 65-year-old with IIIA } \\
\text { clear-cell ovarian carcinoma }\end{array}$ & HTN & 2 & 5 & $\begin{array}{l}\text { Carboplatin/ } \\
\text { gemcitabine }\end{array}$ & Continued & H/o chronic HTN \\
\hline $\begin{array}{l}\text { 3. 80-year-old with IA granulosa } \\
\text { cell tumor }\end{array}$ & HTN & 3 & 3 & Cytoxan & $\begin{array}{l}\text { Held } \times \text { I cycle, } \\
\text { discontinued } \\
\text { after cycle } 5\end{array}$ & H/o chronic HTN \\
\hline $\begin{array}{l}\text { 4. 62-year-old with IIIC ovarian } \\
\text { adenocarcinoma }\end{array}$ & HTN & 4 & 14 & $\begin{array}{l}\text { Carboplatin/ } \\
\text { gemcitabine }\end{array}$ & Discontinued & $\begin{array}{l}\text { Nephrotic syndrome, } \\
\text { h/o chronic HTN }\end{array}$ \\
\hline $\begin{array}{l}\text { 5. } 65 \text {-year-old with } \mathrm{IIICI} \text {, } \\
\text { grade } 2 \text { endometrial } \\
\text { adenocarcinoma }\end{array}$ & Proteinuria & 3 & 25 & Paclitaxel & Discontinued & $\begin{array}{l}\text { H/o chronic HTN, } \\
\text { DM }\end{array}$ \\
\hline $\begin{array}{l}\text { 6. 62-year-old with IIC, grade } 2 \text {, } \\
\text { serous ovarian carcinoma }\end{array}$ & $\begin{array}{l}\text { Fistula } \\
\text { (enterovaginal) }\end{array}$ & NA & 5 & $\begin{array}{l}\text { Liposomal } \\
\text { doxorubicin }\end{array}$ & Discontinued & $\begin{array}{l}\mathrm{H} / \mathrm{o} \text { prior vaginal cuff } \\
\text { radiation }\end{array}$ \\
\hline
\end{tabular}

Abbreviations: HTN, hypertension; DM; diabetes mellitus; NA, not applicable; H/o, history of.

had platinum-sensitive disease. The first Bev-containing and Bev-retreatment regimens are shown in Table 2. Four patients (17.4\%) had a complete response, seven patients (30.4\%) had a partial response, eight patients (34.8\%) had stable disease, and four patients (17.4\%) had progressive disease with the first Bev-containing regimen. During retreatment with Bev, six (26.1\%) patients had a clinical response, and $13(56.5 \%)$ had progressive disease. The four patients (17.4\%) on single-agent Bev had stable disease. Response to the first Bev-containing regimen was not predictive of subsequent response to retreatment with Bev, as three patients (50\%) with clinical response to retreatment had progressive disease on their initial Bev-containing regimen.

The median OS from initial surgery to last contact or death was 63.4 months ( $95 \%$ confidence interval [CI] 40.1-86.6). The median OS periods from diagnosis of recurrence and from retreatment with Bev to the last contact or death were 14.6 months (95\% CI 10.7-18.6) and 9.7 months (95\% CI 2.2-17.3), respectively. The Bev-free interval was also clinically significant in terms of survival. On univariate analysis, there was a statistically significant $(P=0.003)$ improvement in OS from diagnosis of recurrence when the Bev-free interval was greater than 9 months. Those patients survived 24.3 months (95\% CI 4.9-43.7) compared to patients with a Bev-free interval of 9 months or less, who survived 6.8 months (95\% CI 2.1-11.5) (Figure 1). The majority of patients, $73.7 \%$, had received only one chemotherapeutic regimen between initial treatment and retreatment with Bev. There were too few patients receiving two or more interBev chemotherapeutic regimens to draw any conclusions regarding outcome based on this variable. When examining patients who had received only one chemotherapeutic regimen between Bev-containing regimens, those whose Bev-free interval was greater than 9 months lived significantly longer (mean OS 25.6 months \pm 7.6 months) than those whose Bev-free interval was 9 months or less (mean OS 8.6 months \pm 1.9 months) (Figure 2, $P=0.032$ ).

\section{Discussion}

Currently, there are at least eight major clinical trials in EOC that include Bev. Five trials are evaluating its use in recurrent disease, two trials include Bev in primary adjuvant therapy, and one trial is assessing the feasibility of incorporating Bev into neoadjuvant chemotherapy. ${ }^{14}$ Although not the standard of care, the use of Bev in frontline and/or recurrent EOC treatment has been increasing. There is a paucity of data on cumulative treatment-related toxicity and response to $\mathrm{Bev}$ in patients exposed to multiple chemotherapeutic regimens containing this agent. In this study, more than a quarter of patients with recurrent gynecologic malignancy treated with Bev had received more than one Bev-containing regimen. Additionally, almost $75 \%$ of these patients completed their planned treatment regimens without significant Bevassociated toxicity.

The majority of the literature on Bev-associated toxicity comes from trials in nongynecologic malignancy, including colorectal, breast, and non-small-cell lung cancer. ${ }^{15-23}$ The most common toxicities reported include headache, HTN, epistaxis, and proteinuria. ${ }^{24}$ Serious complications are rare, and include fistula formation, arterial thromboembolic events, and hypertensive crisis. Bowel perforation was reported in earlier Bev trials, ${ }^{25}$ and while its rates were slightly higher in the Bev-containing arms of GOG-218 and ICON7, they were still less than $3 \%$ and $1 \%$, respectively. There were no bowel 


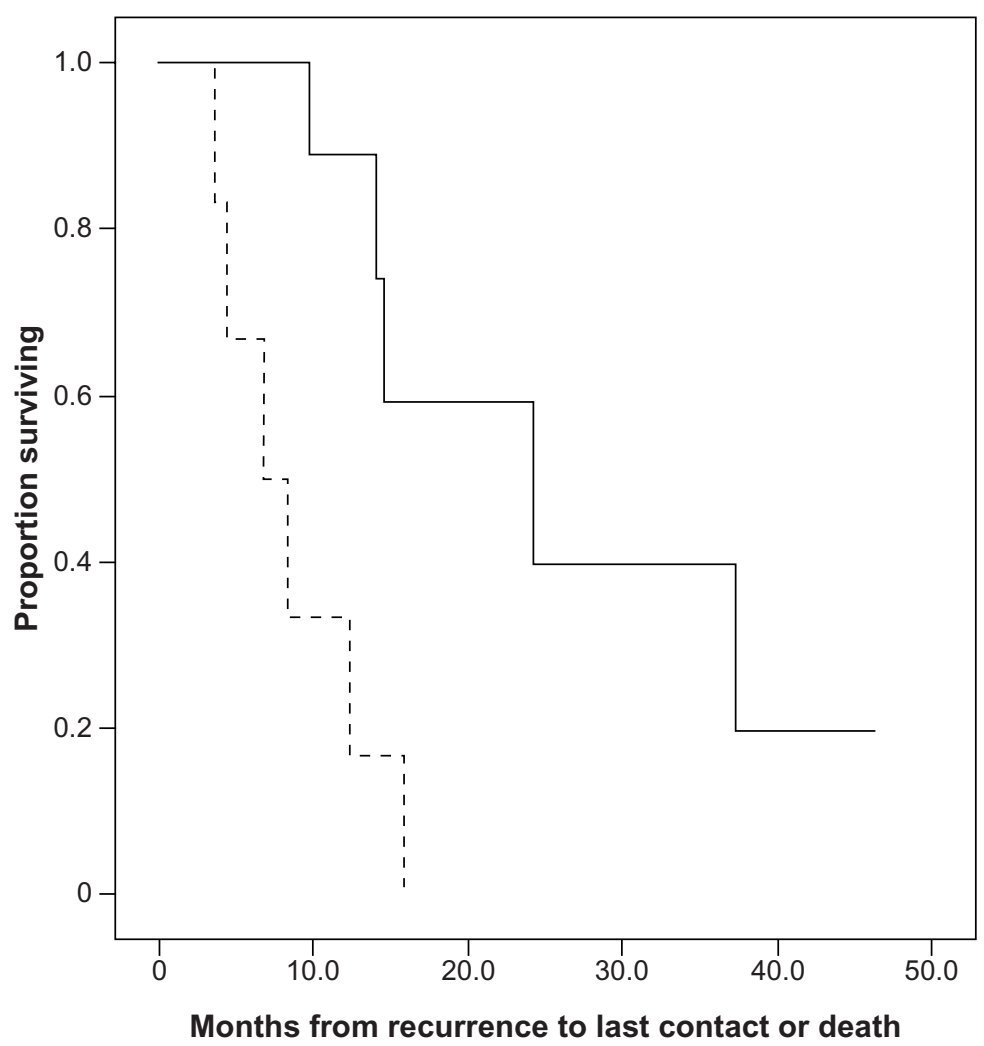

Bevacizumab-free interval

- Less than or equal to

- 9 months

$\neg$ Greater than 9 months

Figure I Overall survival, in months, from recurrence to the last contact or death, based on a bevacizu statistically significant $(P=0.003)$ improvement of 17.5 months when the Bev-free interval was $>9$ months.

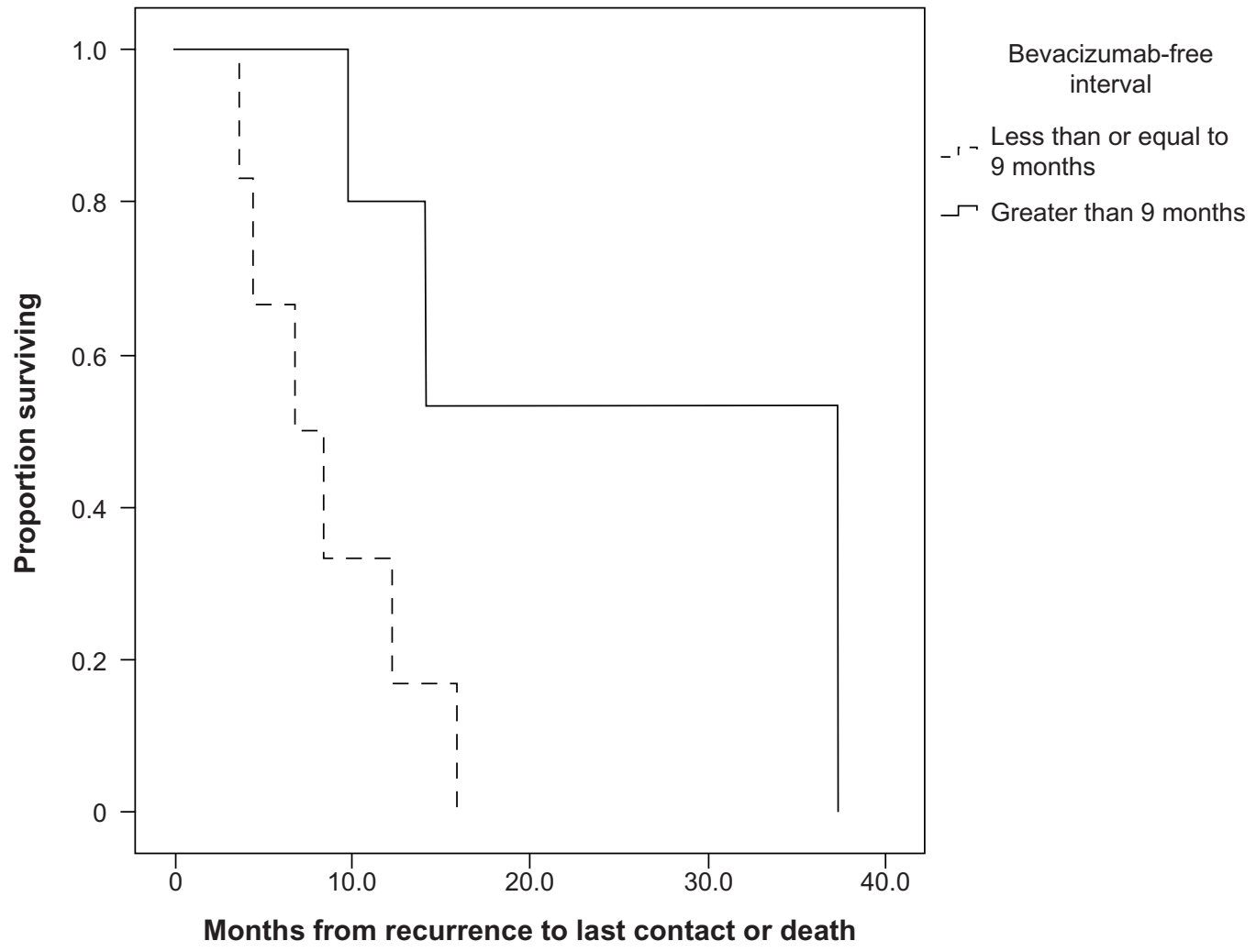

Figure 2 Overall survival, in months, from recurrence to the last contact or death, based on a bevacizumab (Bev)-free interval of $>9$ months or $\leq 9$ months for those patients that had only one inter-Bev chemotherapeutic regimen. The improved survival of 17 months in patients with a Bev-free interval of $>9$ months was statistically significant $(P=0.032)$. 
perforations in our study. Several factors may contribute to the development of bowel perforation, which can have a $50 \%$ mortality rate, such as three or more prior chemotherapy regimens and bowel-wall thickening or bowel obstruction. ${ }^{26}$ Our study demonstrates the tolerability of Bev in patients retreated with this agent, even when the total number of cycles exceeded 30. Only four patients discontinued treatment due to Bev-associated toxicity. Similarly, in a Phase II trial of single-agent Bev in patients with recurrent EOC or primary peritoneal carcinoma, five of $62(8.1 \%)$ patients were treated with 30-35 cycles with acceptable toxicity. ${ }^{27}$ While study populations were similar, the present study in conjunction with the Phase II trial highlights the suitability of Bev in selected patients in the recurrent setting, even with prolonged use.

Although not the standard of care, patients are being treated with Bev maintenance, a practice that some feel is supported by the results of GOG-218. ${ }^{2}$ Others have argued that Bev maintenance should not be considered as a frontline treatment option until biologic markers predictive of response or improved OS benefit have been demonstrated. ${ }^{28}$ Studies in colorectal cancer have not proven tumor VEGF expression, tumor microvessel density, or KRAS and TP53 mutations to be predictive of Bev response. ${ }^{29,30}$ However, there is evidence that in some tumor types single-nucleotide polymorphisms of VEGF may serve as biomarkers to predict outcome and Bev-induced toxicity. ${ }^{31,32}$ Recent clinical trial design allows for continuation of investigational treatments, including Bev, until progression of disease or unacceptable toxicity. ${ }^{1}$ Other agents, such as paclitaxel, have been used for maintenance therapy in patients with advanced-stage EOC. Unfortunately, extended taxane therapy is associated with the development of cumulative toxicity, such as peripheral neuropathy. ${ }^{33}$ Our study did not identify any such cumulative toxicity related to prolonged use of Bev.

McCann et al recently published a retrospective review detailing the clinical outcomes of patients with recurrent EOC who initially experienced a complete response on a Bev-containing regimen. The PFS and OS of patients retreated with Bev were compared against patients that did not have additional Bev-containing regimens. The authors reported a 14-month improvement in PFS in patients retreated with Bev (20 months versus 6 months; $P<0.01$ ). ${ }^{34}$ The improvement in overall survival was not statistically significant. Additionally, $88 \%$ of patients retreated with Bev experienced clinical benefit compared to $50 \%$ of those not retreated with $\mathrm{Bev}(P=0.01)$.

The timing of retreatment with Bev may have a significant impact on outcome, as highlighted by an improvement in
OS with prolongation of the Bev-free interval in our patient population. This finding is similar to published literature that suggests prolongation of the platinum-free interval may improve treatment response and reduce resistance. ${ }^{35-37}$ It has been hypothesized that prolonging the platinum-free interval with the use of non-cross-resistant chemotherapy leads to eradication of resistant clones, thereby improving response to retreatment with platinum. ${ }^{37} \mathrm{~A}$ similar argument can be made for retreatment with Bev. Alternatively, the prolongation in OS related to the Bev-free interval seen in this study may indeed be an indicator of tumor biology, similar to the platinum-free interval and platinum resistance seen during frontline therapy. The secondary aim of this retrospective study was to examine the utility of retreatment with Bev in recurrent gynecologic malignancy. In order to confirm the findings herein, a prospective interventional trial utilizing randomization to control for differing tumor biology would be required.

While not currently a recognized clinical entity, resistance to antiangiogenic agents may play a role in response to treatment with Bev. Several mechanisms have been postulated for the development of resistance. Because antiangiogenic agents work by starving tumors of oxygen and nutrient-rich blood supplied by neovascularization, those tumor cells with the capability of surviving in hypoxic conditions may be selected for with the use of antiangiogenic factors. ${ }^{38}$ Other proangiogenic factors can be upregulated as a result of the downregulation of VEGF, such as placental growth factor, fibroblast growth factor and $\delta$-like ligand $4 .{ }^{39}$ Tumors may also avert the need for neoangiogenesis by co-opting normal vasculature. $^{40}$

Although $42 \%$ of patients in the GOG-170D trial were platinum-resistant, treatment with single-agent Bev produced a $21 \%$ clinical response rate, with an additional $52 \%$ of patients experiencing stable disease. ${ }^{27}$ In this group of patients, a remarkable $40 \%$ had a PFS greater than 6 months. Interestingly, on multivariable analysis, there was no significant association with prior platinum sensitivity or progression, arguing that even patients considered platinum-resistant may experience clinical benefit from treatment with Bev. Similarly, in our patient population, prior response to Bev did not predict subsequent treatment response, and $50 \%$ of those with clinical response had progressed on a prior Bevcontaining regimen. Backes et al reported comparable findings in their patients with recurrent EOC treated with more than one Bev-containing regimen. Almost $20 \%$ of patients that did not respond to initial treatment with Bev responded to a subsequent Bev-containing regimen. ${ }^{41}$ One possible 
explanation for this finding is that Bev may function as a chemosensitizing agent by causing normalization of tumor vasculature, thereby aiding delivery of cytotoxic chemotherapy to the tumor. ${ }^{42}$

The weaknesses of this current study include those that are inherent to all retrospective reviews. Our sample size was relatively small and a variety of cytotoxic chemotherapy regimens were used in the recurrent setting, making it difficult to control for individual treatment regimens' effects on toxicity or response to Bev. Although a substantial percentage of patients were suboptimally debulked, this clinical variable was accounted for in univariate and multivariate analysis. Additionally, almost half of the patients in this study were platinum-resistant or -refractory, which may have impacted clinical response rates. The majority of patients in this study did not receive Bev frontline, and it is unclear what impact, if any, this may have on response to Bev and Bev-associated toxicity in recurrent disease. This study was designed to prove that a larger randomized trial powered to answer questions related to response and outcome in patients retreated with Bev is safe and feasible.

\section{Conclusion}

In conclusion, the utilization of Bev in patients with recurrent gynecologic malignancy who have previously been treated with Bev is safe and feasible, and in many patients will produce prolonged response. The decision to retreat with Bev must be based on several factors, including prior treatment regimens, prior response to treatment, performance status, and baseline medical comorbidities. Similarly, the management of Bev-associated toxicity, specifically the decision to reduce dosage or discontinue treatment, requires assessment of the severity of the adverse event, potential long-term consequences, goals of therapy, and quality of life. Physicians treating women with recurrent disease may consider the addition of Bev to cytotoxic chemotherapy, even if previous regimens included Bev. Future studies should prospectively evaluate the efficacy of this treatment strategy for recurrent disease.

\section{Disclosure}

The authors report no conflicts of interest in this work.

\section{References}

1. Banerjee S, Kaye SB. Gynecological cancer: first-line bevacizumab for ovarian cancer - new standard of care? Nat Rev Clin Oncol. 2012;9(4): 194-196.

2. Burger RA, Brady MF, Bookman MA, et al. Incorporation of bevacizumab in the primary treatment of ovarian cancer. $N$ Engl J Med. 2011;365(26):2473-2483.
3. Perren TJ, Swart AM, Pfisterer J, et al. A phase 3 trial of bevacizumab in ovarian cancer. $N$ Engl J Med. 2011;365(26):2484-2496.

4. European Medicines Agency. Summary of opinion (post authorization): Avastin (bevacizumab). Available from: http://www.ema.europa.eu/ docs/en_GB/document_library/Summary_of_opinion/human/000582/ WC500112811.pdf. Accessed January 9, 2014.

5. Aghajanian C, Blank SV, Goff BA, et al. OCEANS: a randomized, double-blind, placebo-controlled phase III trial of chemotherapy with or without bevacizumab in patients with platinum-sensitive recurrent epithelial ovarian, primary peritoneal, or fallopian tube cancer. J Clin Oncol. 2012;30(17):2039-2045.

6. National Cancer Institute. Protocol development: Common terminology criteria for adverse events. Available from: http://ctep.cancer.gov/ protocolDevelopment/electronic_applications/ctc.htm. Accessed January 9, 2014.

7. Therasse P, Arbuck SG, Eisenhauer EA, et al. New guidelines to evaluate the response to treatment in solid tumors. European Organization for Research and Treatment of Cancer, National Cancer Institute of the United States, National Cancer Institute of Canada. J Natl Cancer Inst. 2000;92(3):205-216.

8. Rustin GJ. Use of CA-125 to assess response to new agents in ovarian cancer trials. J Clin Oncol. 2003;21(Suppl 10):187s-193s.

9. Rosen MA. Use of modified RECIST criteria to improve response assessment in targeted therapies: challenges and opportunities. Cancer Biol Ther. 2010;9(1):20-22.

10. Rustin GJ, Marples M, Nelstrop AE, Mahmoudi M, Meyer T. Use of CA-125 to define progression of ovarian cancer in patients with persistently elevated levels. J Clin Oncol. 2001;19(20):4054-4057.

11. Rustin GJ, Nelstrop AE, Bentzen SM, Piccart MJ, Bertelsen K. Use of tumour markers in monitoring the course of ovarian cancer. Ann Oncol. 1999;10 Suppl 1:21-27.

12. Rustin GJ, Nelstrop AE, McClean P, et al. Defining response of ovarian carcinoma to initial chemotherapy according to serum CA 125. J Clin Oncol. 1996;14(5):1545-1551.

13. Rustin GJ, Nelstrop AE, Tuxen MK, Lambert HE. Defining progression of ovarian carcinoma during follow-up according to CA 125: a North Thames Ovary Group Study. Ann Oncol. 1996;7(4):361-364.

14. National Cancer Institute [website on the Internet]. Available from: http://www.cancer.gov. Accessed January 9, 2014.

15. Allegra CJ, Yothers G, O’Connell MJ, et al. Initial safety report of NSABP C-08: a randomized phase III study of modified FOLFOX6 with or without bevacizumab for the adjuvant treatment of patients with stage II or III colon cancer. J Clin Oncol. 2009;27(20): 3385-3390.

16. Escudier B, Pluzanska A, Koralewski P, et al. Bevacizumab plus interferon alfa-2a for treatment of metastatic renal cell carcinoma: a randomised, double-blind phase III trial. Lancet. 2007;370(9605): 2103-2111.

17. Giantonio BJ, Catalano PJ, Meropol NJ, et al. Bevacizumab in combination with oxaliplatin, fluorouracil, and leucovorin (FOLFOX4) for previously treated metastatic colorectal cancer: results from the Eastern Cooperative Oncology Group Study E3200. J Clin Oncol. 2007;25(12):1539-1544.

18. Hurwitz H, Fehrenbacher L, Novotny W, et al. Bevacizumab plus irinotecan, fluorouracil, and leucovorin for metastatic colorectal cancer. N Engl J Med. 2004;350(23):2335-2342.

19. Miller K, Wang M, Gralow J, et al. Paclitaxel plus bevacizumab versus paclitaxel alone for metastatic breast cancer. $N$ Engl J Med. 2007;357(26):2666-2676.

20. Miller KD, Chap LI, Holmes FA, et al. Randomized phase III trial of capecitabine compared with bevacizumab plus capecitabine in patients with previously treated metastatic breast cancer. J Clin Oncol. 2005;23(4):792-799.

21. Reck M, von Pawel J, Zatloukal P, Ramlau R, Gorbounova V, Hirsh V, et al. Phase III trial of cisplatin plus gemcitabine with either placebo or bevacizumab as first-line therapy for nonsquamous non-small-cell lung cancer: AVAil. J Clin Oncol. 2009;27(8):1227-1234. 
22. Saltz LB, Clarke S, Díaz-Rubio E, et al. Bevacizumab in combination with oxaliplatin-based chemotherapy as first-line therapy in metastatic colorectal cancer: a randomized phase III study. J Clin Oncol. 2008;26(12):2013-2019.

23. Sandler A, Gray R, Perry MC, et al. Paclitaxel-carboplatin alone or with bevacizumab for non-small-cell lung cancer. $N$ Engl J Med. 2006;355(24):2542-2550.

24. Randall LM, Monk BJ. Bevacizumab toxicities and their management in ovarian cancer. Gynecol Oncol. 2010;117(3):497-504.

25. Han ES, Monk BJ. What is the risk of bowel perforation associated with bevacizumab therapy in ovarian cancer? Gynecol Oncol. 2007;105(1): 3-6.

26. Cannistra SA, Matulonis UA, Penson RT, et al. Phase II study of bevacizumab in patients with platinum-resistant ovarian cancer or peritoneal serous cancer. J Clin Oncol. 2007;25(33):5180-5186.

27. Burger RA, Sill MW, Monk BJ, Greer BE, Sorosky JI. Phase II trial of bevacizumab in persistent or recurrent epithelial ovarian cancer or primary peritoneal cancer: a Gynecologic Oncology Group Study. J Clin Oncol. 2007;25(33):5165-5171.

28. Copur MS, Obermiller AM, Ramaekers R. Bevacizumab in ovarian cancer. N Engl J Med. 2012;366(13):1256-1257; author reply 7-8.

29. Ince WL, Jubb AM, Holden SN, et al. Association of k-ras, b-raf, and p53 status with the treatment effect of bevacizumab. J Natl Cancer Inst. 2005;97(13):981-989.

30. Jubb AM, Hurwitz HI, Bai W, et al. Impact of vascular endothelial growth factor-A expression, thrombospondin-2 expression, and microvessel density on the treatment effect of bevacizumab in metastatic colorectal cancer. J Clin Oncol. 2006;24(2):217-227.

31. Schneider BP, Wang M, Radovich M, et al. Association of vascular endothelial growth factor and vascular endothelial growth factor receptor-2 genetic polymorphisms with outcome in a trial of paclitaxel compared with paclitaxel plus bevacizumab in advanced breast cancer: ECOG 2100. J Clin Oncol. 2008;26(28):4672-4678.

32. Schultheis AM, Lurje G, Rhodes KE, et al. Polymorphisms and clinical outcome in recurrent ovarian cancer treated with cyclophosphamide and bevacizumab. Clin Cancer Res. 2008;14(22):7554-7563.
33. Markman M, Liu PY, Moon J, et al. Impact on survival of 12 versus 3 monthly cycles of paclitaxel $\left(175 \mathrm{mg} / \mathrm{m}^{2}\right)$ administered to patients with advanced ovarian cancer who attained a complete response to primary platinum-paclitaxel: follow-up of a Southwest Oncology Group and Gynecologic Oncology Group phase 3 trial. Gynecol Oncol. 2009;114(2):195-198.

34. McCann GA, Smith B, Backes FJ, et al. Recurrent ovarian cancer: is there a role for re-treatment with bevacizumab after an initial complete response to a bevacizumab-containing regimen? Gynecol Oncol. 2012;127(2):362-366.

35. Bookman MA. Extending the platinum-free interval in recurrent ovarian cancer: the role of topotecan in second-line chemotherapy. Oncologist. 1999;4(2):87-94.

36. Kavanagh J, Tresukosol D, Edwards C, et al. Carboplatin reinduction after taxane in patients with platinum-refractory epithelial ovarian cancer. J Clin Oncol. 1995;13(7):1584-1588.

37. Tanguay JS, Ansari J, Buckley L, Fernando I. Epithelial ovarian cancer: role of pegylated liposomal doxorubicin in prolonging the platinum-free interval and cancer antigen 125 trends during treatment. Int J Gynecol Cancer. 2009;19(3):361-366.

38. Kerbel RS. Tumor angiogenesis. N Engl J Med. 2008;358(19): 2039-2049.

39. Crawford Y, Ferrara N. Tumor and stromal pathways mediating refractoriness/resistance to anti-angiogenic therapies. Trends Pharmacol Sci. 2009;30(12):624-630.

40. Bergers G, Hanahan D. Modes of resistance to anti-angiogenic therapy. Nat Rev Cancer. 2008;8(8):592-603.

41. Backes FJ, Richardson DL, McCann GA, et al. Should bevacizumab be continued after progression on bevacizumab in recurrent ovarian cancer? Int J Gynecol Cancer. 2013;23(5):833-838.

42. Jain RK. Normalizing tumor vasculature with anti-angiogenic therapy: a new paradigm for combination therapy. Nat Med. 2001;7(9): 987-989.
OncoTargets and Therapy

\section{Publish your work in this journal}

OncoTargets and Therapy is an international, peer-reviewed, open access journal focusing on the pathological basis of all cancers, potential targets for therapy and treatment protocols employed to improve the management of cancer patients. The journal also focuses on the impact of management programs and new therapeutic agents and protocols on

\section{Dovepress}

patient perspectives such as quality of life, adherence and satisfaction The manuscript management system is completely online and includes a very quick and fair peer-review system, which is all easy to use. Visit http://www.dovepress.com/testimonials.php to read real quotes from published authors. 